The Quality Network for Prison Mental Health Services used the findings from this consultation to produce a national guidance document on planning effective mental healthcare in prisons, which can be accessed for free by all prison mental health teams.

\section{Communication in COVID: a quality improvement project into staff communication with family/carers at New Haven Older Adult Mental Health Inpatient Unit \\ Felicity Jones $^{1 \star}$, Bhavna Khanna ${ }^{1}$, Batool Almoosawi ${ }^{1}$, Alex Humm ${ }^{1}$, Upjeet Mahon ${ }^{2}$ and Rosie Edwards ${ }^{2}$ \\ ${ }^{1}$ Hereford and Worcestershire Health and Care NHS Trust and \\ ${ }^{2}$ University of Birmingham, Medical School \\ ${ }^{*}$ Corresponding author.}

doi: 10.1192/bjo.2021.534

Aims. In the psychiatric care of patients, family involvement is key to recovery. At the New Haven Unit, there have been a number of complaints regarding poor communication and lack of updates given to families during COVID-19.

The aim is to:

To increase the overall satisfaction of the family with the service received for their loved ones

Ensure effective and timely communication of updates to the families, to prevent further complaints, by assigning a member of staff per patient to be the primary individual responsible for family contact

Create an addition to the weekly ward round MDT proforma on 'Carenotes' where communication can be documented

Method. A standardised questionnaire has been sent to the relatives of inpatients at the New Haven Unit. Qualitative data are being collated, which will lead to quantitative statistical analysis of the satisfaction ratings.

Based on the current bed state on the ward at the time of the project all 32 relatives of current inpatients were contacted and 23 agreed to complete the survey which was sent out either by email or post.

The new MDT proforma will be added, which will be used to record actions needed to be taken involving communication and updating family members on a weekly basis. This opportunity to record communication will improve continuity of care and satisfaction amongst family members.

There will be follow-up via a second questionnaire to identify improvement.

Result. The average results of selected categories so far are shown below (still awaiting further responses):

Frequency of updates regarding loved ones $=4.33 / 10$ (10-excellent)

To what degree were your concerns listened to? 7.33/10

Quality of content discussed with staff members $=3.33 / 4$ (4excellent)

Other categories scoring below the expected standard, included awareness of visiting guidelines and questions regarding lasting power of attorney, in which $33.3 \%$ of participants responded either 'no' or 'not sure' respectively.

Questions addressing formalities of introduction and confidentiality through identity confirmation, scored highly.

Conclusion. We are awaiting more survey responses in order to identify additional areas of improvement; however, it is already clear to see that there are areas that would be advanced through structured, assigned reminders via an MDT amendment.

We will also be introducing set dates for conference calls with the families now involving the whole MDT; one within the first week of admission, one after six weeks and one at the point of discharge as a minimum.

\section{North West London New Model of Care Project (NMOC) - improving inpatient mental health care for children and young people}

Dr Jovanka Tolmac ${ }^{1 \star}$, Alun Lewis ${ }^{2}$, Azer Mohammed ${ }^{3}$, Elizabeth Fellow-Smith ${ }^{4}$, Johan Redelinghuys ${ }^{5}$ and Braulio Girelas ${ }^{6}$

${ }^{1}$ Consultant Child and Adolescent Psychiatrist, Harrow CAMHS, Central and North West London NHS Foundation Trust; ${ }^{2}$ NMOC Project manager, West London NHS Trust; ${ }^{3}$ Consultant Child and Adolescent Psychiatrist, Westminster CAMHS, Central and North West London NHS Foundation Trust; ${ }^{4}$ Consultant Child and Adolescent Psychiatrist, West London NHS Trust; ${ }^{5}$ CAMHS Clinical Director, West London Trust and ${ }^{6}$ Clinical Research Fellow, Centre for Psychiatry, Imperial College, Centre for Paediatrics and Child Health, Faculty of Medicine, Imperial College, Honorary Spr, Harrow CAMHS, Central and North West London NHS Foundation Trust ${ }^{*}$ Corresponding author.

\section{doi: 10.1192/bjo.2021.535}

Aims. Specialised inpatient mental health services for children and young people are commissioned and managed by NHS England (NHSE) and provided by NHS as well as independent sector. The access to beds has been managed nationally with young people admitted far from home. There were capacity issues identified in London. To address these concerns, NHSE invited organisations to work in partnership to co-design and establish new models of care. This is one of the first of such projects, set up to manage the budget for children and young people's beds on behalf of NHSE and change the way of managing and monitoring admissions.

Our aims:

To reduce length of inpatient stay

To enable admission of young people as close to home as possible

To improve resource efficiency, capacity and capability of managing young people in crisis in the community.

Method. A number of changes were introduced, including engagement of community and inpatient clinical staff, repatriation to units closer to home and introduction of CRAFT meetings (early review meetings in inpatient units to enable timely and effective discharge planning and support back to local services). The implementation has been closely monitored by the project manager and clinical group, which included representatives from all organisations involved.

Result. After four years, young people are admitted to hospitals closer to home and the length of inpatient stay has decreased by $18 \%$. The number of admissions has decreased by $28 \%$. Out of area occupied beds days have been decreased by $66 \%$.

Significant recurrent budget savings have been achieved. Over the past three years, these savings have been reinvested in developing crisis community support and more specialist community services within CNWL and West London Trust.

Conclusion. There have been considerable benefits of multiple organisations working in partnership to improve patients care. The success of the project has created further opportunities for the development of services which provide safe and effective alternatives to admission (such as crisis services, home treatment teams and specialized community services). In summary, this collaborative model has improved the quality of care and experience for young people and reduced the need for psychiatric admission. 